\title{
PKCE Increases Extracellular Elastin and Fibulin-5/DANCE in Dermal Fibroblasts
}

\author{
Tomoyuki Nishizaki \\ Innovative Bioinformation Research Organization, Kobe, Japan
}

\section{Key Words}

PKC $\bullet$ Elastin • Fibulin-5/DANCE • Elastic fibre $・$ Dermal fibroblast

\begin{abstract}
Background/Aims: In the earlier study, the selective PKC $\varepsilon$ activator DCP-LA increased elastic fibres in the dermis of HR-1 hairless mice. As a process of elastic fibre formation, tropoelastin, an elastin monomer, is secreted into the extracellular space. Secreted tropoelastin is delivered to the microfibrils by fibulin-5/developmental arteries and neural crest epidermal growth factor-like (DANCE) and undergoes self-association. Then, tropoelastin assembles around the microfibrils, growing into elastin and elastic fibres by lysyl oxidase (LOX)- or LOX-like (LOXL)mediated cross-linking. The present study was conducted to understand the mechanism underlying DCP-LA-induced increase in elastin/elastic fibre. Methods: Western blotting, immunocytochemistory, and real-time reverse transcription-polymerase chain reaction (RT$\mathrm{PCR}$ ) were carried out in cultured human dermal fibroblasts. $\mathrm{PKC \varepsilon}$, mammalian target of rapamycin complex (mTOR), and p70 S6 kinase (S6K) were knocked-down by transfecting each siRNA. Results: DCP-LA increased elastin and fibulin-5/DANCE in a treatment time (6-24 h)- and a bell-shaped concentration $(1 \mathrm{nM}-1 \mu \mathrm{M})$-dependent manner in the culture medium of human dermal fibroblasts. DCP-LA markedly increased elastic fibres in the extracellular space of cultured fibroblasts. DCP-LA-induced increase in extracellular elastin and fibulin-5/ DANCE was abolished by a PKC inhibitor or knocking-down PKCE. DCP-LA did not affect expression of mRNAs for tropoelastin and fiblin-5/DANCE in cultured fibroblasts. DCP-LAinduced increase in extracellular elastin and fibulin-5/DANCE was not inhibited by the protein synthesis inhibitor cycloheximide or by knocking-down mTOR and S6K. DCP-LA never increased extracellular elastin in the presence of elastase, that breaks down elastin. An inhibitor of matrix metalloproteinase 9 , that degrades multiple extracellular matrix components including elastin, had no effect on the basal levels and the DCP-LA-induced increase levels of extracellular elastin. Conclusion: The results of the present study indicate that PKCE, activated by DCP-LA, increases elastin and fibulin-5/DANCE in the extracellular space of cultured fibroblasts by the mechanism independent of transcriptional and translational modulation or inhibition of elastolysis.




\section{Cellular Physiology Cell Physiol Biochem 2018;46:291-302 and Biochemistry Published online: March 27, 2018 \begin{tabular}{l|l} 
DOI: 10.1159/000488430 & $\begin{array}{l}\text { c } 2018 \text { The Author(s). Published by S. Karger AG, Basel } \\
\text { www.karger.com/cpb }\end{array}$
\end{tabular} \\ Nishizaki et al.: $\mathrm{PKC} \varepsilon$-Mediated Elastogenesis}

\section{Introduction}

Tissues are composed of cells and extracellular matrix. The extracellular matrix serves as a scaffold to support cells, which includes collagen, elastin, and hyaluronic acid. Elastin is present in the skin, blood vessels, and lung, and regulates structural integrity and elasticity required for mechanical stretching of tissues and organs as a normal function [1]. Tropoelastin, an elastin monomer, is produced in cells. Tropoelastin is secreted into the extracellular space via secretory vesicles, and secreted tropoelastin undergoes self-association under the physiological conditions (coacervation) and assembles around the microfibrils [2]. Lysyl oxidase (LOX) or LOX-like (LOXL) mediates cross-linking of tropoelastin at lysine residues, growing into elastin and elastic fibres [2]. The tropoelastin-binding protein fibulin-5/developmental arteries and neural crest epidermal growth factor-like (DANCE) delivers tropoelastin to the microfibrils, and organizes and links elastic fibres to the cell surface through integrins, to normally function as elastic fibres in tissues and organs [3-5].

Synthesis of tropoelastin is changed with age. Expression of the tropoelastin mRNA and formation of elastic fibre are primarily found within a limited period during development [6]. The tropoelastin synthesis closely correlates to the tropoelastin mRNA levels, and expression of elastin is mainly controlled by the pre-/post-transcriptional and pre-translational mechanisms [7]. In the in vivo experiments using transgenic mice, activity of the human tropoelastin promoter in the skin, which is coupled to the chloramphenicol acetyltransferase (CAT) reporter gene, peaks at 3 months, thereafter returning to the postnatal basal levels [8]. The tropoelastin synthesis in the chick aorta decreases with age in part due to destabilization of the tropoelastin mRNA [9].

Expression of the tropoelastin mRNA is regulated by growth factors and hormones. Transforming growth factor- $\beta$ (TGF- $\beta$ ) upregulates human tropoelastin promoter activity in transgenic mice [10]. TGF- $\beta 1$ increases tropoelastin production by enhancing activity of the tropoelastin promoter or post-transcriptionally stabilizing the tropoelastin mRNA [10-14]. Stabilization of the tropoelastin mRNA is achieved by a variety of factors such as active Smads, phosphatidylcholine-specific phospholipase C, PKC $\delta$, stress-activated protein kinase, and p38 mitogen-activated protein kinase [12, 14]. 17 $\beta$-estradiol increases tropoelastin synthesis by stimulating TGF- $\beta$ signaling [15]. In contrast, TGF- $\beta 1$-induced upregulation of the tropoelastin mRNA is inhibited by tumor necrosis factor- $\alpha$ and interferon- $\gamma$ [10]. Insulin-like growth factor I and interleukin-1 $\beta$ upregulate tropoelastin gene expression $[16,17]$, while vitamin $D$ represses steady-state and functional levels of the tropoelastin mRNA by the post-transcriptional mechanism [18]. Aldosterone induces tropoelastin gene expression and elastic fibre deposition, and the elastogenic effect of aldosterone is enhanced by mineralocorticoid receptor antagonists [19].

In my earlier study, application of the selective PKCe activator 8-[2-(2-pentyl cyclopropylmethyl)-cyclopropyl]-octanoic acid (DCP-LA) to the dorsal skin of HR-1 hairless mice increased elastic fibres in the extracellular matrix of the dermis under the normal conditions and restored ultraviolet B (UVB) irradiation-induced decrease of elastic fibres [20]. DCP-LA was detected in the epidermis and dermis $3 \mathrm{~h}$ after application to the dorsal skin of mice, the concentrations being 0.61 and $0.34 \mathrm{nmol} / \mathrm{mg}$ protein, respectively, which confirms that DCP-LA is permeable to the skin and is capable of exerting an effect in the dermis. The present study was conducted to understand the mechanism underlying DCP-LA-induced increase in elastic fibres. The results show that DCP-LA increases elastin and fibulin-5/DANCE in the extracellular space of cultured human dermal fibroblasts in a PKCE-dependent manner, regardless of transcriptional and translational modulation or inhibition of elastolysis.

\section{Materials and Methods}

\section{Cell culture}

A human dermal fibroblast cell line, which was derived from the dermis of normal human adult skin and cryopreserved at the end of primary culture, was purchased from Lonza (Verviers, Belgium). In the present study, one cell line was used for different experiments. Lonza's human dermal fibroblasts are 


\section{Cellular Physiology Cell Physiol Biochem 2018;46:291-302 \\ \begin{tabular}{l|l|l} 
and Biochemistry Published online:March 27, 2018 & $\begin{array}{l}\text { C } 2018 \text { The Author(s). Published by S. Karger AG, Basel } \\
\text { www.karger.com/cpb }\end{array}$ \\
\hline
\end{tabular}}

Nishizaki et al.: PKC $\varepsilon$-Mediated Elastogenesis

guaranteed through 15 population doublings when using $\mathrm{FGM}^{\mathrm{TM}}-2$ Growth Media and characteristics of dermal fibroblasts are confirmed by morphological observation throughout serial passage. Human dermal fibroblast cells were grown in $\mathrm{FGM}^{\mathrm{TM}}-2$ BulletKit ${ }^{\mathrm{TM}}$ (Lonza) in a humidified atmosphere of 5\% $\mathrm{CO}_{2}$ and $95 \%$ air at $37^{\circ} \mathrm{C}$, and after three to five passages cells were used for experiments.

\section{Quantification of elastin and fibulin-5/DANCE}

Cultured fibroblasts were treated without and with DCP-LA, and then the culture medium was collected. After precipitation with $5 \%(\mathrm{w} / \mathrm{v})$ trichloroacetic acid, proteins were separated by sodium dodecyl sulfate-polyacrylamide gel electrophoresis using a TGX gel (BioRad, Hercules, CA, USA) and transferred to polyvinylidene difluoride membranes. Blotting membranes were blocked with TBS-T [150 mM NaCl, $0.1 \%$ (v/v) Tween-20 and $20 \mathrm{mM}$ Tris, $\mathrm{pH}$ 7.5] containing 5\% (w/v) bovine serum albumin and subsequently incubated with an antibody against elastin (Millipore, Temecula, CA, USA) or fibulin-5 (Sigma, St Louis, MO, USA). Blotting membranes were reacted with a horseradish peroxidase-conjugated goat anti-rabbit IgG antibody. Immunoreactivity was detected with an ECL kit (GE Healthcare, Piscataway, NJ, USA) and visualized using a chemiluminescence detection system (GE Healthcare). The signal density was measured with an ImageQuant software (GE Healthcare).

A series of experiments were performed using cells cultured simultaneously from a tube of cryopreserved cells. After removal of the culture medium, cell layers were lysed and the cell layer protein weight in each well of culture dish was measured using a BCA protein assay kit (Pierce, Rockford, IL, USA). The signal intensity for elastin or fibulin-5/DANCE in the culture medium was normalized by the protein weight of cell layers in each well, and the data were analyzed by calculating the mean $( \pm$ SEM) ratio relative to the basal normalized signal intensity for elastin or fibulin-5/DANCE in the culture medium of fibroblasts untreated with DCP-LA.

\section{Immunocytochemistory}

Cultured fibroblasts were treated without and with DCP-LA. Then, cells were fixed with methanol for $30 \mathrm{~min}$ at $-20^{\circ} \mathrm{C}$ and blocked with $2 \%(\mathrm{w} / \mathrm{v})$ bovine serum albumin in phosphate buffered saline at room temperature. Cells were reacted with an anti-elastin antibody $(1: 100)$ (Millipore) overnight at $4{ }^{\circ} \mathrm{C}$ followed by a goat anti-mouse IgG conjugated with Alexa 488 (Molecular Probes, Eugene, OR, USA) for 60 min at room temperature. After staining with 4',6-diamidino-2-phenylindole (DAPI), fluorescence-labeled cells were visualized with a confocal scanning laser microscope (Axiovert/LSM510; Carl Zeiss, Oberkochen, Germany).

Construction and transfection of the siRNAs

The siRNAs to silence the PKCE-targeted gene with the sequence (5'-GCACUUGCGUUGUCCACAA-3' and 5'-UUGUGGACAACGCAAGUGC-3') and the mammalian target of rapamycin complex (mTOR)-targeted gene with the sequence (5'-GAAUGGUGUCGAAAGUACA-3' and 5'-UGUACUUUCGACACCAU UC-3') were purchased from Santa Cruz Biotechnology and the siRNA to silence the p70 S6 kinase (S6K)-targeted gene with the sequence (5'-GGACAUGGCAGGAGUGUUU-3' and 5'-AAACACUCCUGCCAUGUCC-3') from Ambion (Carlsbad, CA, USA). The negative control (NC) siRNA, which has the scrambled sequence with the GC content and nucleic acid composition same as those for each siRNA was purchased from Ambion. siRNAs were transfected into fibroblasts using a Lipofectamine reagent, and cells were used for experiments $48 \mathrm{~h}$ after transfection.

It was confirmed in the Western blot analysis using antibodies against PKC $\varepsilon$ (BD Biosciences, San Jose, CA, USA), mTOR (Santa Cruz Biotechnology), S6K (Ambion), and $\beta$-actin (Cell Signaling, Beverly, MA, USA) whether each targeted protein was successfully knocked-down.

Real-time reverse transcription-polymerase chain reaction (RT-PCR)

Real-time RT-PCR was carried out using the following primers: for tropoelastin, sense; GGCCATTCCTGGTGGAGTTCC and anti-sense; AACTGGCTTAAGAGGTTTGCCTCCA, for fiblulin-5/DANCE, sense; CTACTCGAACCCCTACTCGAC and anti-sense; TCGTGGGATAGTTTGGAGCTG, and for GAPDH, sense; GACTTCAACAGCGACACCCACTCC and anti-sense; AGGTCCACCACCCTGTTGCTGTAG. Total RNAs of cells were purified by an acid/guanidine/thiocyanate/chloroform extraction method using the Sepasol-RNA I Super kit (Nacalai, Kyoto, Japan). After purification, total RNAs were treated with RNase-free DNase I (2 units) at $37^{\circ} \mathrm{C}$ for $30 \mathrm{~min}$ to remove genomic DNAs, and $10 \mu \mathrm{g}$ of RNAs was resuspended in water. Then, random primers, dNTP, 10x RT buffer, and Multiscribe Reverse Transcriptase were added to an RNA solution and incubated at $25{ }^{\circ} \mathrm{C}$ for $10 \mathrm{~min}$ followed by $37{ }^{\circ} \mathrm{C}$ for $120 \mathrm{~min}$ to synthesize the first-strand cDNA. Realtime RT-PCR was performed using a SYBR Green Realtime PCR Master Mix (Takara Bio) and the Applied 
Biosystems 7900 real-time PCR detection system (ABI, Foster City, CA, USA). Thermal cycling conditions were as follows: first step, $94{ }^{\circ} \mathrm{C}$ for $4 \mathrm{~min}$; the ensuing 40 cycles, $94{ }^{\circ} \mathrm{C}$ for $1 \mathrm{~s}, 65^{\circ} \mathrm{C}$ for $15 \mathrm{~s}$, and $72{ }^{\circ} \mathrm{C}$ for $30 \mathrm{~s}$. The expression level of each mRNA was normalized by that of the GAPDH mRNA.

\section{Statistical analysis}

Statistical analysis was carried out using unpaired $t$-test, Dunnett's test, and analysis of variance (ANOVA) followed by a Bonferroni correction.

\section{Results}

DCP-LA increases extracellular elastin/ elastic fibre and fibulin-5/DNACE

DCP-LA increased elastin in the culture medium of human dermal fibroblasts in a treatment time (1-24 h)-dependent manner, with the maximum at $12 \mathrm{~h}$, and in a bell-shaped concentration $(1 \mathrm{nM}-1 \mu \mathrm{M})$-dependent manner, with the maximum at $100 \mathrm{nM}$ (Fig. 1A,B,D). DCP-LA also increased fibulin-5/DANCE in the culture medium in a treatment time (1-24 h)dependent manner, with the maximum at $1 \mathrm{~h}$, and in a bell-shaped concentration (1 nM-1 $\mu \mathrm{M})$-dependent manner, with the maximum at $100 \mathrm{nM}$ (Fig. 1A,C,E).

In the immunocytochemical analysis, DCP-LA markedly increased elastic fibres in the extracellular space of cultured fibroblasts as compared with those for control (Fig. 2). Collectively, these results indicate that DCP-LA is capable of increasing elastin/elastic fibre and fibulin-5/DANCE in the extracellular space of cultured fibroblasts.

PKCE is a key factor for DCP-LA-induced increase in extracellular elastin and fibulin-5/DNACE

DCP-LA serves as a selective PKC $\varepsilon$ activator $[21,22]$. The basal levels of extracellular elastin were decreased by the PKC inhibitor GF109203X, but not significantly, while the basal levels of extracellular fibulin-5/DANCE were not affected (Fig. 3A,B). DCP-LA-induced increase in extracellular elastin and fibulin-5/ DANCE was abolished by GF109203X (Fig. $3 \mathrm{~A}, \mathrm{~B})$. This indicates that DCP-LA increases extracellular elastin and fibulin-5/DANCE in a PKCE-dependent manner.

To obtain further evidence for this, PKC $\varepsilon$ was knocked-down using the PKC $\varepsilon$ siRNA. Expression of $\mathrm{PKC} \varepsilon$ in cultured fibroblasts was clearly suppressed by transfecting with the PKCE siRNA (Fig. 2C), which confirms successful knock-down of PKCE. The basal levels of extracellular elastin and fibulin-5/DANCE were significantly decreased by knocking-down

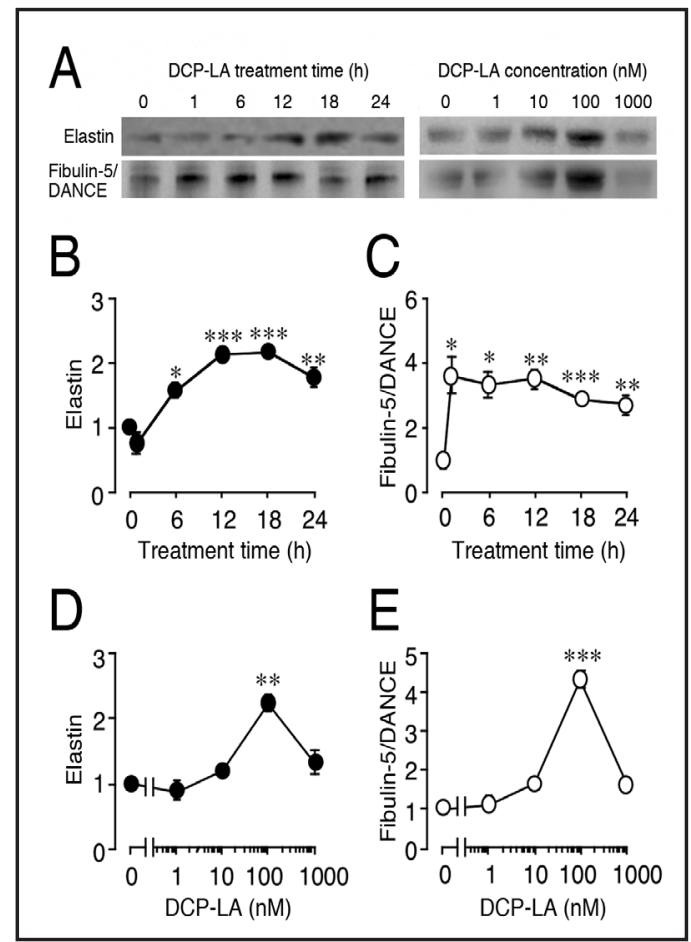

Fig. 1. DCP-LA increases extracellular elastin and fibulin-5/DANCE. Cultured fibroblasts were treated with DCP-LA (100 nM) for periods of time as indicated or DCP-LA at concentrations as indicated for $24 \mathrm{~h}$. Then, Western blotting was carried out in the culture medium using antibodies against elastin and fiblin-5/DANCE. The signal intensity for elastin or fibulin-5/DANCE was normalized by the cell layer protein weight in each well of culture dish. (A) Typical blotting images. (B)(D) Data represent the mean $( \pm$ SEM) ratio relative to basal normalized signal intensity for elastin in the culture medium of fibroblasts untreated with DCP-LA $(n=8$ independent series of experiments). (C)(E) Data represent the mean $( \pm$ SEM) ratio relative to the basal normalized signal intensity for fibulin-5/ DANCE in the culture medium of fibroblasts untreated with DCP-LA ( $\mathrm{n}=8$ independent series of experiments). ${ }^{*} \mathrm{P}<0.05,{ }^{*} \mathrm{P}<0.01,{ }^{* * *} \mathrm{P}<0.001$ as compared with the basal signal intensity, Dunnett's test. 
Fig. 2. DCP-LA increases elastic fibres in the extracellular space. Cultured fibroblasts were treated without (Control) and with DCPLA (100 $\mathrm{nM}$ ) for $24 \mathrm{~h}$, followed by immunocytochemistry using antibodies against elastin and DAPI. Bars, $100 \mu \mathrm{m}$. Note that similar results were obtained with 4 independent series of experiments.

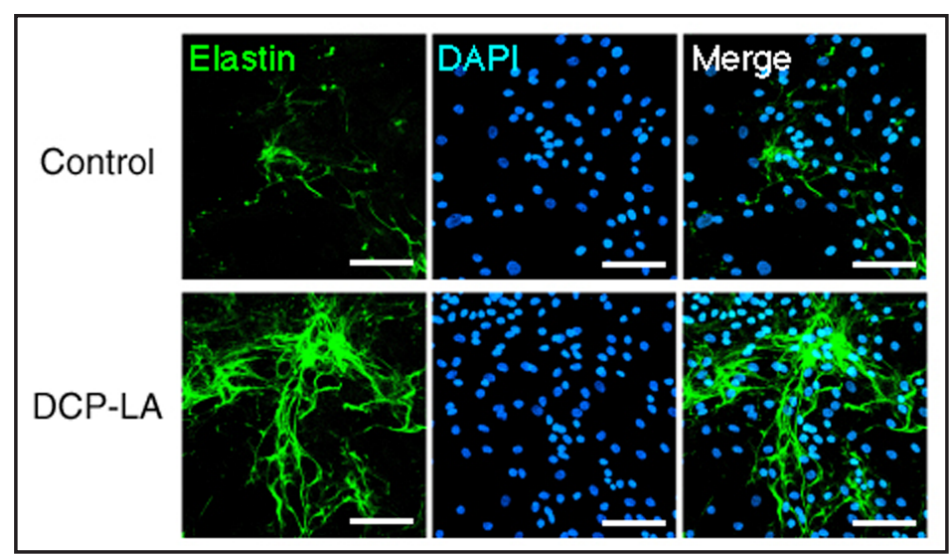

Fig. 3. DCP-LA increases extracellular elastin and fibulin-5/DANCE in a PKCEdependent manner. Cultured fibroblasts were treated with DCP-LA (100 nM) in the presence and absence of GF109203X (100 nM) for 12 h, followed by Western blotting in the culture medium. The signal intensity for elastin or fibulin-5/DANCE was normalized by the cell layer protein weight in each well of culture dish. In the graphs, each column represents the mean $( \pm$ SEM) ratio relative to the basal normalized signal intensity for elastin (A) or fibulin-5/DANCE (B) in the culture medium of fibroblasts untreated with DCPLA $(n=4$ independent series of experiments). $\mathrm{P}$ values, ANOVA followed by a Bonferroni correction. (C) Cultured fibroblasts were transfected

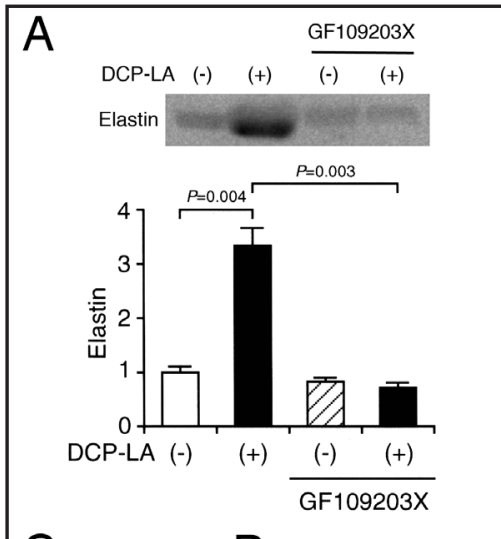

C
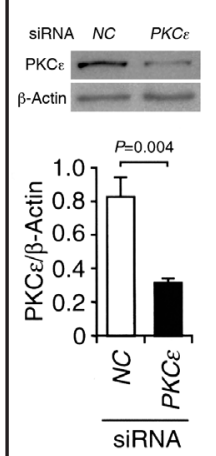

B
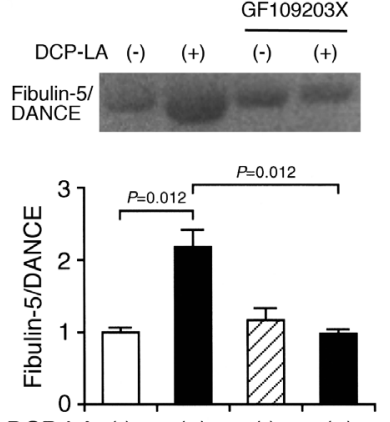

$\operatorname{DCP}-\operatorname{LA}(-) \quad(+) \quad(-) \quad(+)$

GF109203X

E
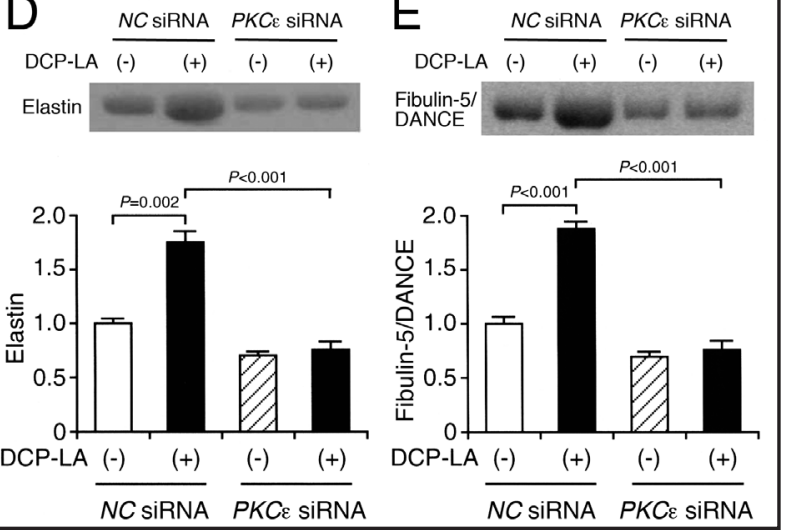

with the negative control (NC) siRNA or the PKC $\varepsilon$ siRNA, and $48 \mathrm{~h}$ later Western blotting was carried out in cell lysates. In the graph, each column represents the mean ( \pm SEM) signal intensity for PKCe normalized by that for $\beta$-actin ( $n=4$ independent series of experiments). $P$ value, unpaired t-test. Cultured fibroblasts with transfected with the NC siRNA or the PKCe siRNA were treated without and with DCP-LA (100 nM) for 12 $\mathrm{h}$, followed by Western blotting in the culture medium. The signal intensity for elastin or fibulin-5/DANCE was normalized by the cell layer protein weight in each well of culture dish. In the graphs, each column represents the mean $( \pm$ SEM) ratio relative to the basal normalized signal intensity for elastin (D) or fibulin-5/ DANCE (E) in the culture medium of fibroblasts transfected with the NC siRNA without DCP-LA treatment $(\mathrm{n}=4$ independent series of experiments). $\mathrm{P}$ values, ANOVA followed by a Bonferroni correction.

PKCE $(P=0.014$ and 0.021 as compared with the levels in cells transfected with the NC siRNA, respectively; ANOVA followed by a Bonferroni correction) (Fig. 3D,E). This suggests that PKC $\mathrm{E}$ plays 
a role to increase the basal levels of extracellular elastin and fibulin-5/DANCE. DCP-LA-induced increase in extracellular elastin and fibulin-5/DANCE was abrogated by knocking-down PKC $\varepsilon$ (Fig. 3D,E). This provides direct evidence that PKC $\varepsilon$ is a key factor for DCP-LA-induced increase in extracellular elastin and fibulin-5/DANCE.

\section{DCP-LA increases extracellular elastin and fibulin-5/DNACE without affecting transcription and translation}

DCP-LA had no effect on the mRNA levels for tropoelastin and fibulin-5/DANCE in cultured fibroblasts (Fig. 4A,B). This indicates that DCP-LA-induced increase in extracellular elastin and fibulin-5/DANCE is not due to enhanced transcription of the tropoelastin and fibulin-5/DANCE genes.

The basal levels of elastin and fibulin-5/DANCE in the culture medium of fibroblasts untreated with DCP-LA were obviously reduced by the protein synthesis inhibitor cycloheximide (CHX) (Fig. 5A,B). Amazingly, DCP-LA increased extracellular elastin and fibulin-5/DANCE still in the presence of CHX, to an extent similar to that in the absence of CHX (Fig. 5A,B). This indicates that DCP-LA-induced increase in extracellular elastin and fibulin-5/DANCE is not due to an enhancement in the synthesis of tropoelastin and fibulin-5/DANCE.

mTOR functions in two distinct multiprotein complexes such as mTOR complex 1 (mTORC1) and mTOR complex 2 (mTORC2), and mTORC1 initiates protein synthesis by targeting S6K [23, 24]. DCP-LA is capable of activating Akt through PKCE-mediated phosphorylation of Akt [25]. Akt activates Rheb, a Ras family GTPase, by inhibiting tuberous sclerosis complex 2 (TSC2), a GTPaseactivating protein (GAP) toward Rheb, followed by activation of mTORC1 [24]. Akt, alternatively, activates mTORC1 by inhibiting proline-rich Akt substrate of $40 \mathrm{kDa}$ (PRAS40), an mTORC1 nega-

Fig. 4. DCP-LA does not affect the mRNA levels for tropoelastin and fibulin-5/DANCE. Cultured fibroblasts were treated with DCP-LA (100 nM) for periods of time as indicated, followed by real-time RT-PCR. In the graphs, each column represents the mean $( \pm$ SEM) mRNA level for tropoelastin (A) or fibulin-5/DANCE (B) normalized by that for GAPDH $(n=4$ independent series of experiments).

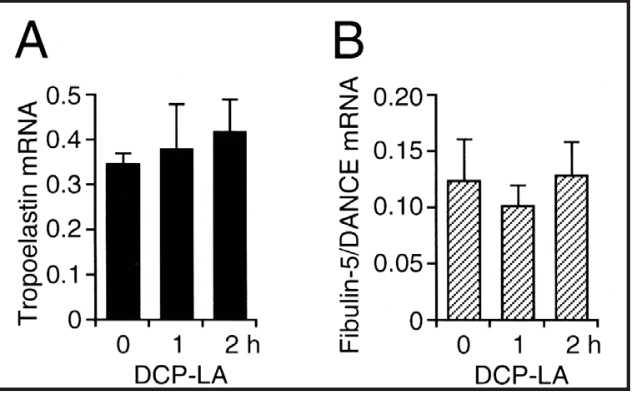

Fig. 5. DCP-LA-induced increase in extracellular elastin and fibulin-5/DANCE is not due to enhancement of each protein synthesis. Cultured fibroblasts were incubated in the absence and presence of CHX (5 $\mu \mathrm{M})$ for $6 \mathrm{~h}$ prior to 12 -h treatment without and with DCP-LA (100 nM), followed by Western blotting in the culture medium. The signal intensity for elastin or fibulin-5/ DANCE was normalized by the cell layer protein weight in each well of culture dish.

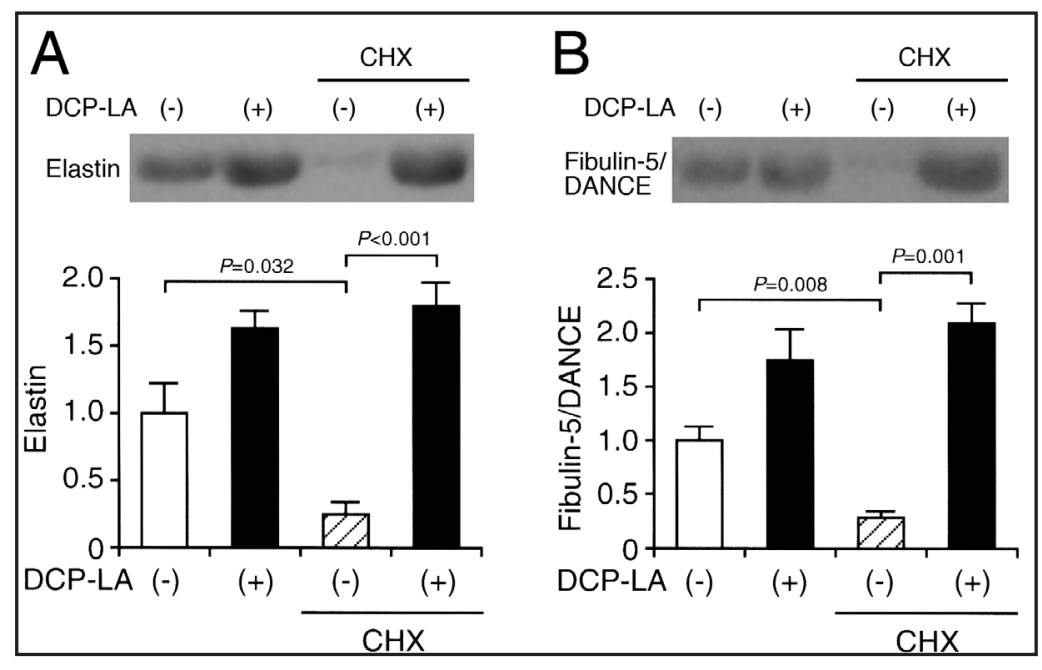
In the graphs, each column represents the mean $( \pm S E M)$ ratio relative to the basal normalized signal intensity for elastin (D) or fibulin-5/DANCE (E) in the culture medium of fibroblasts untreated with DCP-LA in the absence of CHX ( $n=4$ independent series of experiments). P values, ANOVA followed by a Bonferroni correction. 
Fig. 6. $M T O R$ is not implicated in DCP-LAinduced increase in extracellular elastin and fibulin-5/DANCE. Cultured fibroblasts were transfected with the negative control (NC) siRNA or the MTOR siRNA, and $48 \mathrm{~h}$ later Western blotting was carried out in cell lysates. In the graph, each column represents the mean $( \pm$ SEM) signal intensity for mTOR

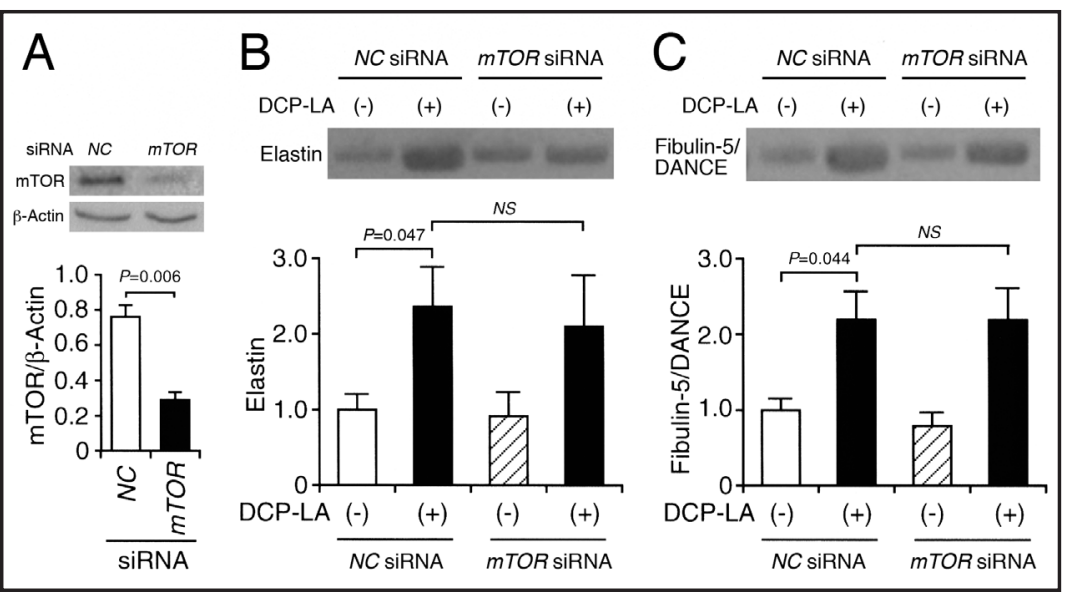
normalized by that for $\beta$-actin ( $\mathrm{n}=4$ independent series of experiments). $\mathrm{P}$ value, unpaired $\mathrm{t}$-test. Cultured fibroblasts with transfected with the NC siRNA or the mTOR siRNA were treated without and with DCP-LA (100 nM) for $12 \mathrm{~h}$, followed by Western blotting in the culture medium. The signal intensity for elastin or fibulin-5/DANCE was normalized by the cell layer protein weight in each well of culture dish. In the graphs, each column represents the mean ( \pm SEM) ratio relative to the basal normalized signal intensity for elastin (B) or fibulin-5/ DANCE (C) in the culture medium of fibroblasts transfected with the NC siRNA without DCP-LA treatment $(n=4$ independent series of experiments). P values, ANOVA followed by a Bonferroni correction. NS, not significant.

Fig. 7. S6K is not implicated in DCP-LA-induced increase in extracellular elastin and fibulin-5/ DANCE. (A) Cultured fibroblasts were transfected with the negative control (NC) siRNA or the S6K siRNA, and 48 h later Western blotting was carried out in cell lysates. In the graph, each column represents the mean ( \pm SEM) signal in-

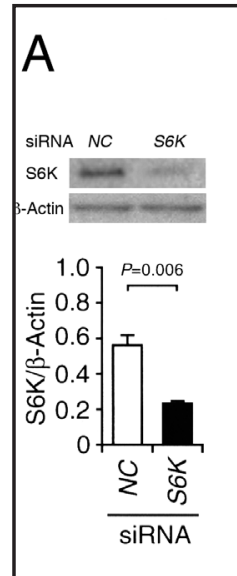

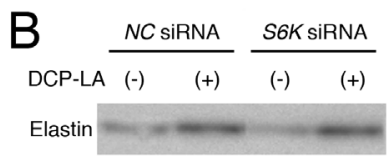
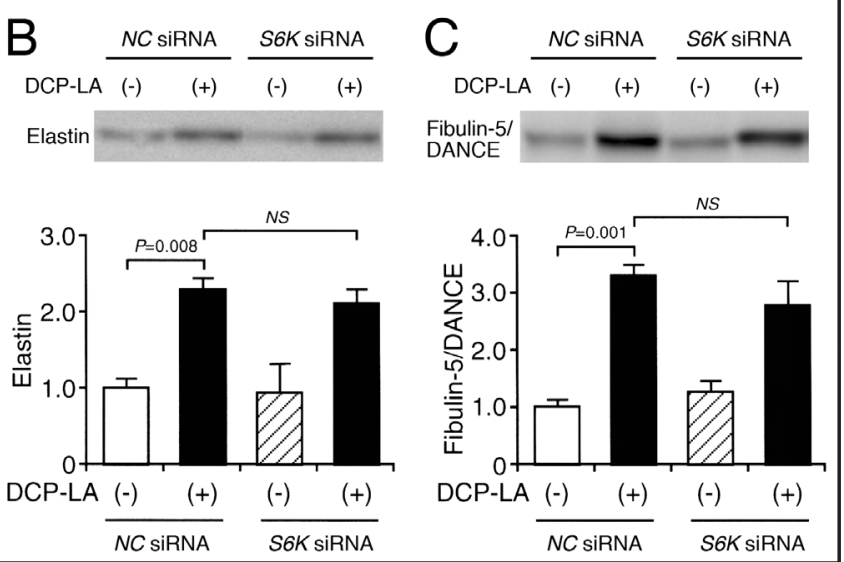
tensity for S6K normalized by that for $\beta$-actin ( $\mathrm{n}=4$ independent series of experiments). $\mathrm{P}$ value, unpaired $\mathrm{t}$-test. Cultured fibroblasts with transfected with the NC siRNA or the S6K siRNA were treated without and with DCP-LA (100 $\mathrm{nM}$ ) for $12 \mathrm{~h}$, followed by Western blotting in the culture medium. The signal intensity for elastin or fibulin-5/DANCE was normalized by the cell layer protein weight in each well of culture dish. In the graphs, each column represents the mean $( \pm$ SEM) ratio relative to the basal normalized signal intensity for elastin (B) or fibulin-5/DANCE (C) in the culture medium of fibroblasts transfected with the NC siRNA without DCP-LA treatment ( $\mathrm{n}=4$ independent series of experiments). $\mathrm{P}$ values, ANOVA followed by a Bonferroni correction. NS, not significant.

tive regulator [24]. DCP-LA, therefore, could stimulate protein synthesis through Akt-mediated mTORC1 activation.

To address this point, mTOR (Fig. 6A) or S6K (Fig. 7A) was knocked-down by transfecting with each siRNA into cultured fibroblasts. The basal levels and DCP-LA-induced increase levels of extracellular elastin were reduced by knocking-down mTOR (Fig. 6B) or S6K, but not significantly (Fig. 7B). mTOR deficiency reduced the basal levels of extracellular fibulin-5/DANCE, but 


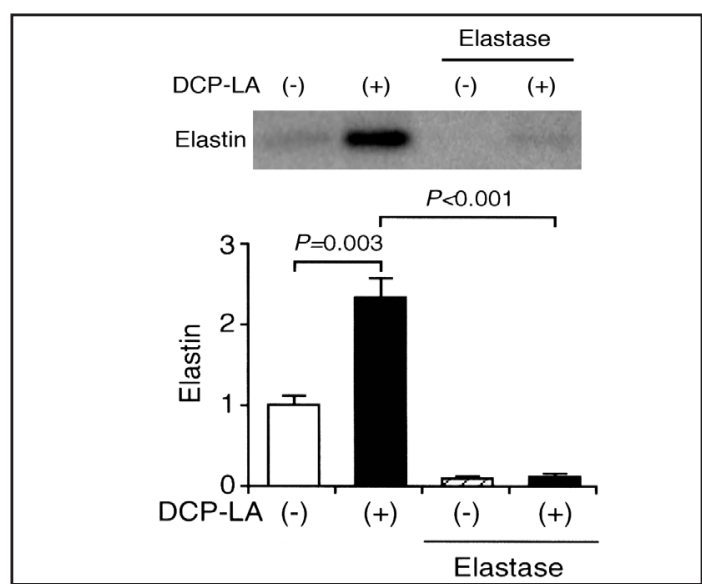

Fig. 8. DCP-LA-induced increase in extracellular elastin is abrogated by elastase. Cultured fibroblasts were treated without and with DCP-LA (100 nM) in the absence and presence of elastase (10 U) for 12 $\mathrm{h}$, followed by Western blotting in the culture medium. The signal intensity for elastin was normalized by the cell layer protein weight in each well of culture dish. In the graphs, each column represents the mean $( \pm$ SEM) ratio relative to the basal normalized signal intensity for elastin without treatment with DCP-LA fin the absence of elastase $(n=4$ independent series of experiments). P values, ANOVA followed by a Bonferroni correction.

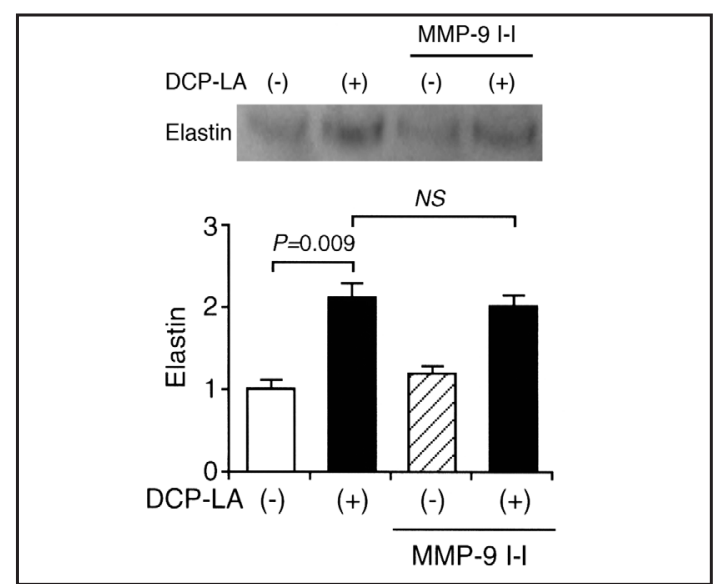

Fig. 9. DCP-LA-induced increase in extracellular elastin is not due to inhibition of elastin degradation. Cultured fibroblasts were treated without and with DCP-LA (100 nM) in the absence and presence of MMP-9I-I $(5 \mu \mathrm{M})$ for $12 \mathrm{~h}$, followed by Western blotting in the culture medium. The signal intensity for elastin was normalized by the cell layer protein weight in each well of culture dish. In the graphs, each column represents the mean $( \pm$ SEM) ratio relative to the basal normalized signal intensity for elastin in the culture medium of fibroblasts untreated with DCP-LA in the absence of MMP-9I-I ( $n=4$ independent series of experiments). P value, ANOVA followed by a Bonferroni correction. NS, not significant.

not significantly, without affecting the DCP-LA-induced increase levels (Fig. 6C). S6K deficiency reduced the DCP-LA-induced increase levels of extracellular fibulin-5/DANCE, but not significantly, without affecting the basal levels (Fig. 7C). Collectively, these results indicate that DCP-LAinduced increase in extracellular elastin and fibulin-5/DANCE is not due to an enhancement in the synthesis of tropoelastin and fibulin-5/DANCE through an Akt/mTORC1/S6K pathway.

\section{DCP-LA-induced increase in extracellular elastin is not due to inhibition of elastolysis}

My final attempt was to examine whether DCP-LA-induced increase in extracellular elastin is due to inhibition of elastolysis.

Elastase, that breaks down elastin, apparently degraded elastin in the culture medium of fibroblasts (Fig. 8). DCP-LA-induced increase in extracellular elastin was not found in the presence of elastase (Fig. 8). This indicates that DCP-LA increases extracellular elastin by the mechanism independent of direct inhibition of elastase.

Matrix metalloproteinase 9 (MMP-9) is a metalloproteinase that degrades multiple extracellular matrix components including elastin, gelatin, collagens III, IV, V, and XI, nidogen-1, and vitronectin. If DCP-LA-induced increase in extracellular elastin is due to inhibition of MMP-9, then MMP-9 inhibitor should exhibit an effect similar to DCP-LA. MMP-9 inhibitor I (MMP-9I-I) increased extracellular elastin by only a little, but the increase did not reach the levels of the DCPLA effect (Fig. 9). Moreover, MMP-9I-I had no effect on DCP-LA-induced increase in extracellular elastin (Fig. 9). These results indicate that DCP-LA increases extracellular elastin, regardless of inhibition of MMP-9.

\section{Discussion}

In the present study, the selective PKC $\varepsilon$ activator DCP-LA increased elastin and fibulin-5/ DANCE in the culture medium of human dermal fibroblasts. The effect of DCP-LA was abolished by a KARGER 


\section{Cellular Physiology Cell Physiol Biochem 2018;46:291-302 \\ and Biochemistry Published online: March 27, $2018 \quad \begin{aligned} & \text { DOI: 10.1159/000488430 } \\ & \begin{array}{l}\text { c) } 2018 \text { The Author(s). Published by S. Karger AG, Basel } \\ \text { ww.karger.com/cpb }\end{array}\end{aligned}$ \\ Nishizaki et al.: PKC $\varepsilon$-Mediated Elastogenesis}

PKC inhibitor or by knocking-down PKCE. These results indicate that DCP-LA increases extracellular elastin and fibulin-5/DANCE in a PKCE-dependent manner. DCP-LA also increased elastic fibres in the extracellular space of fibroblasts, suggesting that DCP-LA promotes formation of elastic fibres. One might claim that DCP-LA-induced increase in elastic fibres in the immunocytochemical analysis seems to be more than DCP-LA-induced increase (approximately 2.5-fold) in elastin in the Western blot analysis. The reason is presently unknown. The immunocytochemical images of elastic fibres shown here were ones in the restricted area of extracellular space per one culture dish and the density of elastic fibres was not homogeneous in the whole extracellular space. Moreover, elastin that constitutes elastic fibres in the immunocytochemical images was not quantified. The quantity of elastin shown in the immunocytochemical images, therefore, may not properly reflect the quantity of elastin obtained in the Western blot analysis. Anyway, it is the case that DCP-LA has the potential to increase elastin/elastic fibre and fibulin-5/DANCE in the extracellular space of cultured human dermal fibroblasts.

The elastin monomer tropoelastin, that is produced in cells, is secreted into the extracellular matrix and undergoes self-association [2]. Fibulin-5/DANCE binds to and delivers secreted tropoelastin to the microfibrils [3-5]. Formation of elastin and elastic fibres is achieved by LOX- or LOXL-mediated cross-linking of tropoelastin $[2,26,27]$. Notably, expression of LOX is upregulated through a PKC/MEK/MAPK-dependent signal transduction pathway [28] and secretion of LOX is induced by PKC [29]. This raises the possibility that DCP-LA might upregulate LOX/LOXL expression and stimulate LOX/LOXL secretion by activating PKCE. Taken together, PKCE, activated by DCP-LA, might stimulate secretion of tropoelastin and fibulin-5/DANCE and promote formation of elastin/elastic fibre under the influence of LOX or LOXL.

Lines of evidence have shown that tropoelastin synthesis is reinforced by enhancing activity of the tropoelastin promoter or post-transcriptionally stabilizing the tropoelastin mRNA [1014]. The mRNA levels for tropoelastin and fibulin-5/DANCE in cultured dermal fibroblasts were not affected by DCP-LA. This rules out the possibility that DCP-LA increases extracellular elastin and fibulin-5/DANCE by upregulating expression of the mRNAs for tropoelastin and fibulin-5/ DANCE. It remains an open question whether DCP-LA upregulates expression of the mRNA for LOX or LOXL.

Tropoelastin synthesis, alternatively, is also enhanced by the pre-translational mechanism [7]. In the present study, DCP-LA increased extracellular elastin and fibulin-5/DANCE still in the presence of the protein synthesis inhibitor CHX, although the basal levels of those proteins were obviously reduced. This rules out the possibility that DCP-LA increases extracellular elastin and fibulin-5/DANCE by enhancing synthesis of tropoelastin and fibulin-5/DANCE.

PKCE, activated by DCP-LA, is capable of directly activating Akt [25]. Akt initiates protein synthesis through an mTORC1/S6K pathway $[23,24]$. The effect of DCP-LA on the extracellular levels of elastin and fibulin-5/DANCE was not cancelled by knocking-down mTOR or S6K. This rules out the possibility that DCP-LA increases extracellular elastin and fibulin-5/DANCE by enhancing synthesis of tropoelastin and fibulin-5/DANCE through an Akt/mTORC1/S6K pathway. Overall, these results allow drawing a conclusion that DCP-LA-induced increase in extracellular elastin and fibulin-5/DANCE is not due to an enhancement in the synthesis of tropoelastin and fibulin-5/DANCE. Whether DCP-LA stimulates synthesis of LOX/LOXL protein, however, is presently unknown.

MicroRNA-214 is shown to increase expression of the mRNAs and proteins for elastin (tropoelastin), fibulin-5/DANCE, and LOX by targeting mitofusin-2 in the process of fibroblast differentiation of adipose-derived mesenchymal stem cells during pelvic floor dysfunction in Sprague Dawley rats with birth trauma [30]. In the present study, DCP-LA did not affect expression of the mRNAs and fibulin-5/DANCE. This would rule out the possibility of the interaction between DCP-LA (PKCE) and microRNA-214.

DCP-LA never increased extracellular elastin in the presence of elastase, that breaks down elastin. This suggests that DCP-LA has no potential to directly inhibit elastase. MMP-9 also degrades multiple extracellular matrix components including elastin. The MMP-9 inhibitor MMP9I-I had no effect on the basal levels and the DCP-LA-induced increase levels of extracellular elastin. This suggests that DCP-LA has no potential to directly inhibit MMP-9. DCP-LA increased 


\section{Cellular Physiology Cell Physiol Biochem 2018;46:291-302

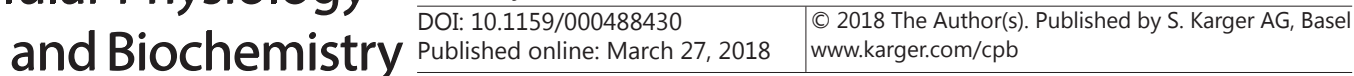 \\ Nishizaki et al.: PKC $\varepsilon$-Mediated Elastogenesis}

extracellular elastin in a PKCE-dependent manner. DCP-LA permeates the cell membrane and activates PKC in cells [31]. This denies direct interaction between intracellular PKC $\varepsilon$ and extracellular MMP-9, further supporting the notion that DCP-LA does not directly inhibit MMP-9. Then, one would wonder whether PKCE, activated by DCP-LA, downregulates expression of MMP9, causing suppression of elastolysis, to increase extracellular elastin. The fact that MMP-9I-I did not mimic the effect of DCP-LA, however, would rule out this possibility. DCP-LA, thus, appears to increase extracellular elastin, regardless of inhibition of elastolysis.

The major question to be addressed is how DCP-LA increases elastin and fibulin-5/DANCE in the extracellular space of cultured human dermal fibroblasts in a PKCE-dependent manner. Studies suggest a pool of extracellular tropoelastin that is ultimately retained as an insoluble polymer in the extracellular matrix $[32,33]$. This implies that a portion of the extracellular tropoelastin pool remains in the medium prior to cross-linking. DCP-LA might maximize the pool in the extracellular matrix that is available for LOX/LOXL-mediated cross-linking. PKCE, activated by DCP-LA, however, could not directly modulate the pool in the extracellular matrix, since PKC $\varepsilon$ exists in cells. A plausible explanation for this is that PKC $\varepsilon$ might stimulate secretion of tropoelastin and fibulin-5/DANCE from cells. Secreted fibulin-5/DANCE might bind to and deliver secreted tropoelastin to not only the microfibrils but the extracellular pool, where tropoelastin is retained. Subsequently, LOX or LOXL might promote cross-linking of tropoelastin retained in the extracellular pool, causing an increase in elastin and elastic fibre.

Accumulating studies have shown that DCP-LA stimulates vesicular release of neurotransmitters such as glutamate, dopamine, serotonin, and $\gamma$-aminobutyric acid in a PKC $\varepsilon$ dependent manner [21, 22, 34, 35]. Moreover, DCP-LA promotes vesicular exocytosis of $\alpha 7$ ACh receptor and increases cell surface localization of the receptor at the presynaptic terminals in a PKC $\varepsilon$-dependent manner [36, 37]. Very interestingly, I have found that DCP-LA promotes PKC $\varepsilon$ dependent vesicular exocytosis of $\alpha 7$ ACh receptor under the influence of $N$-ethylmaleimidesensitive factor (NSF) bearing regulation of vesicular traffic. Consequently, PKC $\varepsilon$, activated by DCP-LA, in the light of the fact that a tropoelastin-containing vesicle exists [2], might stimulate vesicular secretion of tropoelastin, possibly fibulin-5/DANCE as well, by targeting NSF. To prove this hypothesis, further experiments need to be carried out.

Elastic fibres decrease with aging or by UV exposure, responsible for wrinkle formation. In the earlier study, application of DCP-LA to the dorsal skin of HR-1 hairless mice increased the density of elastic fibres in the extracellular matrix of the dermis under the normal conditions [20]. UVB irradiation extremely decreased the density of elastic fibres, but DCP-LA restored UVB irradiation-induced decrease in the density of elastic fibres to the levels same as or higher than the levels under the normal conditions without UVB irradiation. Epidermal hyperplasia also causes wrinkle formation as well as reduction of elastic fibres [38]. UVB irradiation highly thickened the epidermis in the skin of HR-1 hairless mice, and DCP-LA significantly prevented UVB-induced epidermal hyperplasia [20]. These results, taken together with the present results, raise the possibility that DCP-LA has the potential to protect the skin from wrinkle formation by UV exposure or to revive the elasticity of the skin.

\section{Conclusion}

The results of the present study show that PKCE, activated by DCP-LA, increases elastin and fibulin-5/DANCE in the extracellular space of cultured human dermal fibroblasts, without affecting synthesis of tropoelastin and fibulin-5/DANCE or degradation of elastin. This may provide fresh insight into the actions of PKC linked to DCP-LA on elastogenesis.

\section{Abbreviations}

DANCE (Developmental arteries and neural crest epidermal growth factor-like); RT-PCR (Real-time reverse transcription-polymerase chain reaction); LOX (lysyl oxidase); LOXL (LOXlike); mTOR (Mammalian target of rapamycin complex); S6K (p70 S6 kinase); NC (Negative control); CAT (Chloramphenicol acetyltransferase); TGF- $\beta$ (Transforming growth factor- $\beta$ ); DCP- 


\section{Cellular Physiology Cell Physiol Biochem 2018;46:291-302

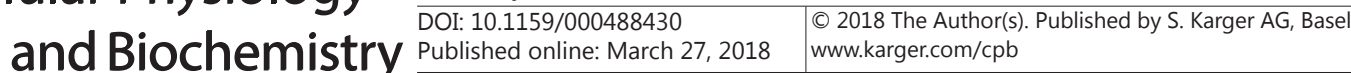 \\ Nishizaki et al.: PKC $\varepsilon$-Mediated Elastogenesis}

LA (8-[2-(2-Pentyl-cyclopropylmethyl)-cyclopropyl]-octanoic acid); UVB (Ultraviolet B); DAPI (4',6-Diamidino-2-phenylindole); ANOVA (Analysis of variance); CHX (Cycloheximide); mTORC (mTOR complex); TSC2 (Tuberous sclerosis complex 2); GAP (GTPase-activating protein); PRAS40 (Proline-rich Akt substrate of $40 \mathrm{kDa}$ ); MMP-9 (Matrix metalloproteinase 9); MMP-9I-I (MMP-9 inhibitor I); NSF ( $N$-Ethylmaleimide-sensitive factor).

\section{Acknowledgements}

I have no sponsorship or funding arrangements relating to my research and I have no competing financial interests.

\section{References}

1 Li DY, Brooke B, Davis EC, Mecham RP, Sorensen LK, Boak BB, Eichwald E, Keating MT: Elastin is an essential determinant of arterial morphogenesis. Nature 1998;393:276-280.

-2 Vrhovski B, Weiss AS: Biochemistry of tropoelastin. Eur J Biochem 1998;258: 1-18.

-3 Yanagisawa H, Davis EC, Starcher BC, Ouchi T, Yanagisawa M, Richardson JA, Olson EN: Fibulin-5 is an elastin-binding protein essential for elastic fibre development in vivo. Nature 2002;415:168-171.

4 Nakamura T, Lozano PR, Ikeda Y, Iwanaga Y, Hinek A, Minamisawa S, Cheng CF, Kobuke K, Dalton N, Takada Y, Tashiro K, Ross Jr J, Honjo T, Chien KR: Fibulin-5/DANCE is essential for elastogenesis in vivo. Nature 2002;415:171-175.

-5 Hirai M, Ohbayashi T, Horiguchi M, Okawa K, Hagiwara A, Chien KR, Kita T, Nakamura T: Fibulin-5/ DANCE has an elastogenic organizer activity that is abrogated by proteolytic cleavage in vivo. J Cell Biol 2007;176:1061-1071.

6 Parks WC, Secrist H, Wu LC, Mecham RP: Developmental regulation of tropoelastin isoforms. J Biol Chem 1988;263:4416-4423.

7 Sephel GC, Buckley A, Davidson JM: Developmental initiation of elastin gene expression by human fetal skin fibroblasts. J Invest Dermatol 1987;88:732-735.

8 Hsu-Wong S, Katchman SD, Ledo I, Wu M, Khillan J, Bashir MM, Rosenbloom J, Uitto J: Tissue-specific and developmentally regulated expression of human elastin promoter activity in transgenic mice. J Biol Chem 1994;269:18072-18075.

-9 Johnson DJ, Robson P, Hew Y, Keeley FW: Decreased elastin synthesis in normal development and in longterm aortic organ and cell cultures is related to rapid and selective destabilization of mRNA for elastin. Circ Res 1995;74:1107-1113.

10 Katchman SD, Hsu-Wong S, Ledo I, Wu M, Uitto J: Transforming growth factor- $\beta$ up-regulates human elastin promoter activity in transgenic mice. Biochem Biophys Res Commun 1994;203:485-490.

11 Kähäri VM, Olsen DR, Rhudy RW, Carrillo P, Chen YQ Uitto J: Transforming growth factor- $\beta$ up-regulates elastin gene expression in human skin fibroblasts. Evidence for post-transcriptional modulation. Lab Invest 1992;66:580-588.

$\checkmark 12$ Kucich U, Rosenbloom JC, Abrams WR, Bashir MM, Rosenbloom J: Stabilization of elastin mRNA by TGF- $\beta$ : initial characterization of signaling pathway. Am J Respir Cell Mol Biol 1997;17:10-16.

13 McGowan SE, Jackson SK, Olson PJ, Parekh T, Gold LI: Exogenous and endogenous transforming growth factors- $\beta$ influence elastin gene expression in cultured lung fibroblasts. Am J Respir Cell Mol Biol 1997;17:25-35.

14 Kucich U, Rosenbloom JC, Abrams WR, Rosenbloom J: Transforming growth factor- $\beta$ stabilizes elastin mRNA by a pathway requiring active Smads, protein Kinase C- $\delta$, and p38. Am J Respir Cell Mol Biol 2002;26:183-188.

15 Son ED, Lee JY, Lee S, Kim MS, Lee BG, Chang IS, Chung JH: Topical application of 17ß-estradiol increases extracellular matrix protein synthesis by stimulating TGF- $\beta$ signaling in aged human skin in vivo. J Invest Dermatol 2005;124:1149-1161.

16 Wolfe BL, Rich CB, Goud HD, Terpstra AJ, Bashir M, Rosenbloom J, Sonenshein GE, Foster JA: Insulin-like growth factor-I regulates transcription of the elastin gene. J Biol Chem 1993;268:12418-12426.

-17 Mauviel A, Chen YQ, Kähäri VM, Ledo I, Wu M, Rudnicka L, Uitto J: Human recombinant interleukin-1 $\beta$ upregulates elastin gene expression in dermal fibroblasts. J Biol Chem 1993;268:6520-6524. 


\section{Cellular Physiology Cell Physiol Biochem 2018;46:291-302 \begin{tabular}{ll|l} 
and Biochemistry & $\begin{array}{l}\text { DOI: 10.1159/000488430 } \\
\text { Published online: March 27, } 2018\end{array}$ & $\begin{array}{l}\text { @ } 2018 \text { The Author(s). Published by S. Karger AG, Basel } \\
\text { www.karger.com/cpb }\end{array}$ \\
\hline
\end{tabular}}

Nishizaki et al.: PKCE-Mediated Elastogenesis

18 Pierce RA, Kolodziej ME, Parks WC: 1, 25-dihydroxyvitamin $\mathrm{D}_{3}$ represses tropoelastin expression by a posttranslational mechanism J Biol Chem 1992;267: 11593-11599.

19 Mitts TF, Bunda S, Wang Y, Hinek A: Aldosterone and mineralocorticoid receptor antagonists modulate elastin and collagen deposition in human skin. J Invest Dermatol 2010;130:2396-2406.

20 Nishizaki T: DCP-LA revives UV irradiation-induced elastic fiber degradation and epithelial hyperplasia. Int J Biol Pharm Res 2016;7:150-153.

-21 Kanno T, Yamamoto H, Yaguchi T, Hi R, Mukasa T, Fujikawa H, Nagata T, Yamamoto S, Tanaka A, Nishizaki T: The linoleic acid derivative DCP-LA selectively activates PKC- $\varepsilon$, possibly binding to the phosphatidylserine binding site. J Lipid Res 2006;47:1146-1156.

-22 Shimizu T, Kanno T, Tanaka A, Nishizaki T: $\alpha, \beta$-DCP-LA selectively activates PKC- $\varepsilon$ and stimulates neurotransmitter release with the highest potency among 4 diastereomers. Cell Physiol Biochem 2011;27:149-158.

23 Magnuson B, Ekim B, Fingar DC: Regulation and function of ribosomal protein S6 kinase (S6K) within mTOR signalling networks. Biochem J 2012;441:1-21.

24 Zhou H, Huang S: The complexes of mammalian target of rapamycin. Curr Protein Pept Sci 2010;11:409424.

25 Kanno T, Tsuchiya A, Tanaka A, Nishizaki T: Combination of PKCe activation and PTP1B inhibition effectively suppresses $A \beta$-induced GSK-3 $\beta$ activation and Tau phosphorylation. Mol Neurobiol 2016;53:4787-4797.

26 Noblesse E, Cenizo V, Bouez C, Borel A, Gleyzal C, Peyrol S, Jacob MP, Sommer P, Damour O: Lysyl oxidaselike and lysyl oxidase are present in the dermis and epidermis of a skin equivalent and in human skin and are associated to elastic fibers. J Invest Dermatol 2004;122:621-630.

27 Sato F, Seino-Sudo R, Okada M, Sakai H, Yumoto T, Wachi H: Lysyl oxidase enhances the deposition of tropoelastin through the catalysis of tropoelastin molecules on the cell surface. Biol Pharm Bull 2017;40:1646-1653.

28 Smith-Mungo L, Kagan HM. PKC-MEK-MAPK-dependent signal transduction pathway mediates the stimulation of lysyl oxidase expression by serum and PDGF in rat aortic smooth muscle cells. J Cell Biochem 2002;85:775-784.

-29 Eun SY, Ko YS, Park SW, Chang KC, Kim HJ. P2Y, nucleotide receptor-mediated extracellular signal-regulated kinases and protein kinase $\mathrm{C}$ activation induces the invasion of highly metastatic breast cancer cells. Oncol Rep 2015;34:195-202.

30 Wu J, Li J, Chen WK, Liu S, Liu JH, Zhang JS, Fang KW: MicroRNA-214 affects fibroblast differentiation of adipose-derived mesenchymal stem cells by targeting mitofusin-2 during pelvic floor dysfunction in SD rats with birth trauma. Cell Physiol Biochem 2017;42:1870-1887.

-31 Kanno T, Tsuchiya A, Shimizu T, Mabuchi M, Tanaka A, Nishizaki T: DCP-LA activates cytosolic PKC $\varepsilon$ by interacting with the phosphatidylserine binding/associating sites Arg50 and Ile89 in the C2-like domain. Cell Physiol Biochem 2015;37:193-200.

-32 Baldwin AK, Simpson A, Steer R, Cain SA, Kielty CM: Elastic fibres in health and disease. Expert Rev Mol Med 2013;15:e8.

-33 Sato F, Wachi H, Ishida M, Nonaka R, Onoue S, Urban Z, Starcher BC, Seyama Y: Distinct steps of crosslinking, self-association, and maturation of tropoelastin are necessary for elastic fiber formation. J Mol Biol 2007;369:841-851.

34 Yamamoto S, Kanno T, Nagata T, Yaguchi T, Tanaka A, Nishizaki T: The linoleic acid derivative FR236924 facilitates hippocampal synaptic transmission by enhancing activity of presynaptic $\alpha 7$ acetylcholine receptors on the glutamatergic terminals. Neuroscience 2005;130:207-213.

35 Kanno T, Yaguchi T, Yamamoto S, Yamamoto H, Fujikawa H, Nagata T, Tanaka A, Nishizaki T: 8-[2-(2-Pentylcyclopropylmethyl)-cyclopropyl]-octanoic acid stimulates GABA release from interneurons projecting to CA1 pyramidal neurons in the rat hippocampus via pre-synaptic $\alpha 7$ acetylcholine receptors. J Neurochem 2005;95:695-702.

-36 Kanno T, Tanaka A, Nishizaki T: Linoleic acid derivative DCP-LA stimulates vesicular transport of $\alpha 7$ ACh receptors towards surface membrane. Cell Physiol Biochem 2012;30:75-82.

37 Kanno T, Tsuchiya A, Tanaka A, Nishizaki T: The linoleic acid derivative DCP-LA increases membrane surface localization of the $\alpha 7 \mathrm{ACh}$ receptor in a protein 4.1N-dependent manner. Biochem J 2013;450:303309.

-38 Matsuda M, Hoshino T, Yamakawa N, Tahara K, Adachi H, Sobue G, Maji D, Ihn H, Mizushima T: Suppression of UV-induced wrinkle formation by induction of HSP70 expression in mice. J Invest Dermatol 2013;133:919-928. 